\title{
Correspondence
}

To the Editors

\section{Clinico-haematological profile of children with vitamin B12 deficiency anaemia}

Sri Lanka Journal of Child Health, 2021; 50(1): 178-179

DOI: http://dx.doi.org/10.4038/sljch.v50i1.9427

(Key words: Clinico-haematological profile, children, vitamin B12 deficiency, anaemia)

The article by Chauhan et $\mathrm{al}^{1}$ describing clinicohematological profile of children with Vitamin B12 deficiency anaemia concisely describes a very pertinent child health problem in South East Asia. However, there is an issue with sample size calculation which requires clarification.

In this cross-sectional study, authors have assumed $\mathrm{P}=50 \%$ to get maximum possible sample size, absolute precision of $6 \%$ and non-response rate of $20 \%$. Using these assumptions, the sample size comes out to be 320 using openEpi online calculator $^{2}$ or standard formula for sample size calculation $^{3}$, instead of 120 as suggested by authors.

Also, the authors have assumed that $50 \%$ of women will have adequate health literacy while making assumption of $\mathrm{P}=50 \%$. The relevance of this assumption in this study requires further description.

\section{References}

1. Chauhan V, Chaudhary RC, Nage S. Clinico-haematological profile of children with vitamin B12 deficiency anaemia. Sri Lanka Journal of Child Health. 2020 Dec $5 ; 49(4): 320-4$.

2. OpenEpi - Toolkit Shell for Developing New Applications [Internet]. [cited 2020 Dec 5]. Available from: https://www.openepi.com/SampleSize/SS Propor.htm

3. Charan J, Biswas T. How to Calculate Sample Size for different study designs in medical research? Indian Journal of Psychological Medicine 2013; 35(2):1216.

\section{Manish Kumar}

Associate Professor of Paediatrics

All India Institute of Medical Sciences

Gorakhpur, India

Correspondence: singh.manish.15@gmail.com

https://orcid.org/0000-0002-3282-0498

Open Access Article published under the Creative $\underline{\text { Response by authors of article }}$

To the Editors,

This is in response to the query raised in relation to the above paper.We thank and appreciate Dr Manish Kumar for his keen observation and pointing out the mistake.We agree that the sample size calculated as per the formula mentioned is different from what was taken in our study. This is actually a human error, a missed typing mistake. The formula used for sample size calculation is detailed as follows:

Sample size formula with desired error of margin:

$$
\mathbf{N}=\frac{Z_{\alpha / 2}{ }^{2} \times \mathbf{P}(1-P)}{d^{2}}
$$

$\mathrm{Z}_{\alpha / 2}$ is the level of significance at $5 \%$ i.e. $95 \%$

Confidence interval- 1.96

P-Prevalence of megaloblastic anaemia $=$ $18 \%=0.18$ (as per previous Indian studies) $)^{1,2,3}$

d- Desired error of margin $=7 \%=0.07$

$$
\begin{gathered}
\mathbf{N}=\underline{\mathbf{1 . 9 6}^{2} \times \mathbf{0 . 1 8} \times(\mathbf{1 - 0 . 1 8})} \\
\mathbf{0 . 0 7 ^ { \mathbf { 2 } }}=115.71
\end{gathered}
$$

Thus Sample size was taken as 120

By assuming non response rate $=10 \%$

$$
\mathrm{n}=\frac{10 \times 120}{100}=12
$$

Final Sample size $=120-12=108$

Hence 110 patients were included in the study

At the inception of this study, during protocol preparation we calculated the sample size using the formula mentioned in the paper.

We did a pilot study and realized that the large sample size calculated cannot be completed given the limited resources and the limited time frame. Hence we recalculated the sample size (as mentioned above) with the trade off between limited statistical power and resource limitation at disposal. It was overlooked and remained uncorrected in the final manuscript due to human error and we really apologize for the same. Regarding the second point that is $50 \%$ assumption 
of women literacy rate which was mentioned with the previous formula we agree, that it is of no significance for the sample size calculation in this study.

Finally,we again thank Dr Manish Kumar for his comments and appreciate his keen observation and acumen. With this reply we present the correct formula used for sample size calculation in our paper.We further state, small sample size as a limitation of our study and hence recommend future studies with larger sample size on this topic to further enhance the knowledge.

\section{References}

1. Dalal RJ, Udani PM, Parekh JG. Megaloblastic anaemia in infancy and childhood. Indian Pediatrics 1969; 6: 25562.
2. Gomber S, Kumar S, Rusia S, et al. Prevalence and aetiology of nutritional anaemias in early childhood in an urban slum. Indian Journal of Medical Research 1998; 107: 269-73.

3. Ghai OP. Aetiology of anaemias in infancy and childhood (in New Delhi). Indian Journal of Child Health 1956; 95(10):6.

With regards,

\section{Richa Chaudhary}

Associate Professor, Department of Paediatrics JNMC, Waedha

Maharashtra, India 